\title{
富锂层状氧化物正极材料研究进展
}

\author{
陈宇方 ${ }^{1}$, 李宇杰 ${ }^{2}$, 郑春满 ${ }^{2}$, 谢 凯 ${ }^{2}$, 陈重学 ${ }^{3}$
}

(1. 国防科学技术大学 指挥军官基础教育学院, 长沙 410073 ; 2. 国防科学技术大学 航天科学与工程学院, 长沙 410073; 3. 武汉大学 动力与机械学院, 武汉 430072)

摘 要: 富锂层状氧化物正极材料 $\left(x \mathrm{Li}_{2} \mathrm{MnO}_{3} \cdot(1-x) \mathrm{LiMO}_{2}\left(\mathrm{M}=\mathrm{Ni}_{y} \mathrm{Mn}_{z} \mathrm{Co}_{1-y-z} \ldots\right)\right.$ 理论容量高、价格低廉, 是新一代锂 离子电池正极材料的候选之一。本文概述了该正极材料的结构, 分析了其在电化学活化过程与循环过程中结构的演 变, 探讨了结构变化对正极材料电化学性能的影响规律, 并概括了目前针对该类正极材料电化学性能提升所开展 的离子掺杂和表面改性的研究工作，展望了未来富锂层状氧化物正极材料的发展方向。

关 键 词: 富锂层状氧化物; 活化和循环过程; 晶相结构演变; 表面改性; 离子掺杂; 综述 中图分类号: TM911 文献标识码: A

\section{Research Development on Lithium Rich Layered Oxide Cathode Materials}

\author{
CHEN Yu-Fang ${ }^{1}$, LI Yu-Jie ${ }^{2}$, ZHENG Chun-Man $^{2}$, XIE Kai $^{2}$, CHEN Zhong-Xue $^{3}$
}

(1. College of Basic Education, National University of Defense Technology, Changsha 410073, China; 2. College of Aerospace Science and Engineering, National University of Defense Technology, Changsha 410073, China; 3. School of Power and Mechanical Engineering, Wuhan University, Wuhan 430072, China)

\begin{abstract}
Lithium rich layered oxide cathode materials $\left(x \mathrm{Li}_{2} \mathrm{MnO}_{3} \cdot(1-x) \operatorname{LiMO}\left(\mathrm{M}=\mathrm{Ni}_{y} \mathrm{Mn}_{z} \mathrm{Co}_{1-y-z} \ldots\right)\right.$ are one of the candidate cathode materials for the new generation of lithium ion batteries because of the high theoretical capacity and low cost. In this paper, the structure of the cathode materials and the structure activation and evolution within initial charge-discharge and the cycling process were outlined, the effect of structure evolution on electrochemical evolution of the Li-rich cathode materials were further investigated. And, the current works of ion doping and surface modification on the cathode materials to enhance electrochemical properties were summarized and the development trend of different lithium rich layered oxide cathode materials was described.
\end{abstract}

Key words: lithium rich layered oxide; activated and cycled process; structure transform; surface modification; cation doping; review

能源是当今世界最为关注发展方向之一, 太 阳能和风能等可再生能源的开发利用已成为时代 主题 ${ }^{[1]}$ 。锂离子二次电池作为可再生能源系统的重 要组成单元, 在满足需求的同时实现丰富的功能 化应用。富锂层状氧化物正极材料由两种或两种以 上晶相构成, 具有 $250 \mathrm{mAh} / \mathrm{g}$ 以上可逆循环容量,
是高容量锂离子二次电池中最具研究与应用前景 的正极材料体系之一。

本文通过总结近年来富锂层状氧化物正极材料 的主要研究成果, 分析探讨这类材料在活化和循环中 晶相结构和电化学性能演变规律, 并在此基础上对这 类正极材料结构改性等相关研究进行分析和讨论。 


\section{1 富锂层状氧化物正极材料的晶相结 构特性}

富锂层状氧化物正极材料结构中包含单斜晶相, 其中 $\mathrm{Li}$ 和 $\left[\mathrm{Li}_{1 / 3} \mathrm{Mn}_{2 / 3}\right]$ 分层排布, 故这一结构也可表 示为 $\left\{\left(\mathrm{Li}_{1 / 2}\right)_{2 \mathrm{c}}\left(\mathrm{Li}_{1}\right)_{4 \mathrm{~h}}\right\}_{\text {interslab }}\left\{\left(\mathrm{Li}_{1 / 2}\right)_{2 \mathrm{~b}}\left(\mathrm{Mn}_{1}\right)_{4 \mathrm{~g}}\right\}_{\text {slab }}\left(\mathrm{O}_{1}\right)_{4 \mathrm{i}}\left(\mathrm{O}_{2}\right)_{8 \mathrm{j}}$ 的形式 ${ }^{[2-3]}$ 。Strobel ${ }^{[4]}$ 和 Massarotti ${ }^{[5]}$ 等分别利用单晶 和粉末 $X$ 射线衍射将其空间群确定为 $\mathrm{C} 2 / \mathrm{m}$ 。

高容量富锂层状氧化物正极材料通常由单斜晶 相 $\mathrm{Li}_{2} \mathrm{MnO}_{3}$ 和层状六方晶相 $\mathrm{LiMO}_{2}(\mathrm{M}=\mathrm{Ni}, \mathrm{Co}, \mathrm{Mn}$ 等 $)$ 构成 ${ }^{[6]}$ (如图 1 所示)。其中, 理想层状六方晶相中两离 子层完全分层排布，而常规合成方法得到的层状六方 晶相中两层会出现 $2 \%$ 20\%离子混排(主要是 $\mathrm{Ni} / \mathrm{Li}$

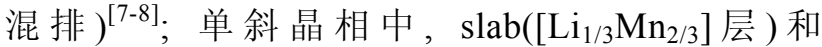
interslab(Li 层)相互堆积(图 1(b) $)^{[9]}$, 堆积顺序会使 单斜晶相出现不同程度缺陷, 改变结构的衍射特 性 $^{[10-11]}$ (图 2(b)所示)。

单斜晶相中, $\mathrm{Li}$ 层和 $\mathrm{Li}-\mathrm{T}^{\mathrm{M}}$ 层沿 $c$ 轴方向的排布 规整度越高, $20^{\circ} \sim 25^{\circ}$ 衍射峰宽越小, $I_{(110)} / I_{(020)}$ 比值 越高, C $2 / \mathrm{m}$ 对称性越低 ${ }^{[11]}$ 。富锂层状氧化物正极材 料的 XRD 图谱包含层状六方晶相衍射峰和 $20^{\circ} \sim 25^{\circ}$ 较弱衍射峰。元素含量和比例、热处理温度和过程 均可以调整材料结构中晶胞常数 ${ }^{[11-13]}$ 。 (a)

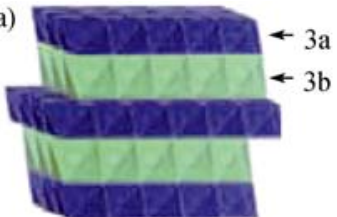

(b)

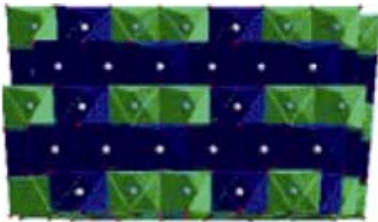

图 1 层状六方晶相与单斜晶相的过渡金属离子和锂离子占 位示意图((a)层状六方晶相; (b)单斜晶相 $)^{[7,9]}$

Fig. 1 Scheme of crystal structure and cation occupancy of transition metal cation and lithium ions in layered rohomohedral phase and monoclinic structure

(a) Layered rohomohedral phase; (b) Monoclinic phase $\mathrm{e}^{[7,9]}$ (a)

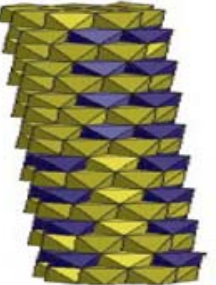

(b)

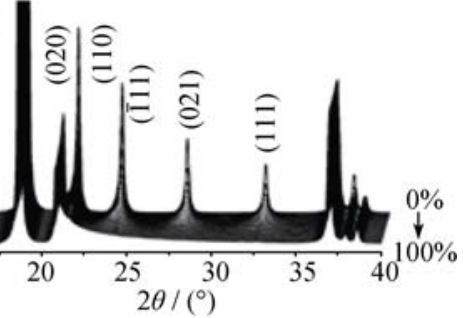

图 $2 \mathrm{Li}_{2} \mathrm{MnO}_{3}$ 晶体结构的堆积位错示意图和不同位错对应的 $\mathrm{XRD}$ 图谱((a)离子堆积方式; (b)不同位错的 XRD 拟合图谱 $)^{[2,11]}$ Fig. 2 Scheme of stacking fault and XRD patterns for various stacking fault in $\mathrm{Li}_{2} \mathrm{MnO}_{3}$

(a) Ionics stacking; (b) XRD simulation pattern of $\mathrm{Li}_{2} \mathrm{MnO}_{3}$ with various stacking fault $)^{[2,11]}$

\section{2 富锂层状氧化物正极材料的活化与 循环过程}

$\mathrm{Li}_{2} \mathrm{MO}_{3}(\mathrm{M}=\mathrm{Ti}, \mathrm{Mn})$ 的早期研究表明，含 $\mathrm{Li}_{2} \mathrm{MO}_{3}$ 的正极材料 $4.3 \mathrm{~V}$ 以上(低于 $\mathrm{Li}_{2} \mathrm{MO}_{3}$ 活化电 压)具有更高容量和良好循环稳定性 ${ }^{[14-15]}$ 。Numata 等 ${ }^{[14]}$ 将 $\mathrm{LiCoO}_{2}$ 与 $\mathrm{Li}_{2} \mathrm{MnO}_{3}$ 复合, 实现 $\mathrm{Li}_{1-x} \mathrm{CoO}_{2}$ 中 $x>0.5$ 锂离子的可逆脱嵌。层状六方晶相和 $\mathrm{Li}_{2} \mathrm{MnO}_{3}$ 构成的正极材料在高于 $\mathrm{Li}_{2} \mathrm{MnO}_{3}$ 活化电压循环时表 现出高容量特性。这类正极材料在 $2 \sim 5.0 \mathrm{~V}$ 循环，可 逆循环容量高于 $250 \mathrm{mAh} / \mathrm{g}^{[16]}$ 。

\section{1 富锂层状氧化物正极材料的活化过程}

富锂层状氧化物的活化机理研究报道较多, 由 于 $\mathrm{Li}_{2} \mathrm{MnO}_{3}$ 中 $\mathrm{Mn}$ 为 +4 价, 在小于 $5 \mathrm{~V}$ 充电条件下 难以将 $\mathrm{Mn}$ 氧化至 +5 价或更高价态 ${ }^{[17]}$, 故认为 $\mathrm{Li}_{2} \mathrm{MnO}_{3}$ 中锂脱出具有其他电荷补偿机制。后续研 究发现，这类正极材料的活化过程中会出现部分氧 从晶格中脱出 ${ }^{[17-18]}$, 发生如式(1)中所示反应，从而 使这类正极材料具有高可逆容量。

$$
\mathrm{Li}_{2} \mathrm{MnO}_{3} \rightarrow 2 \mathrm{Li}^{+}+\mathrm{MnO}_{2}^{-}+\mathrm{O}
$$

理论上 $\mathrm{Li}_{2} \mathrm{MnO}_{3}$ 中锂离子全部脱出可释放的容 量为 $458 \mathrm{mAh} / \mathrm{g}^{[19]}$ 。实际上, 在 $2.0 \sim 5.0 \mathrm{~V}$ 首次充放 电容量分别为 $383 \mathrm{mAh} / \mathrm{g}$ 和 $208 \mathrm{mAh} / \mathrm{g}^{[16]}$, 这一数 值与截至电压相关, $\mathrm{Li}_{2} \mathrm{MnO}_{3}$ 在 $2.0 \sim 4.5 \mathrm{~V}$ 首次充电 容量仅为 $75 \mathrm{mAh} / \mathrm{g}^{[20]}$ 。

富锂层状氧化物 $0.3 \mathrm{Li}_{2} \mathrm{MnO}_{3} \cdot 0.7 \mathrm{LiNi}_{0.5} \mathrm{Mn}_{0.5} \mathrm{O}_{2}$ 首 次充电过程可分为两个部分 ${ }^{[21]}$ : 电压低于 $4.45 \mathrm{~V}$ 时, 锂离子脱出由过渡金属离子氧化实现电荷补偿; 电压 高于 $4.45 \mathrm{~V}$ 时, 过渡金属离子氧化至 +4 价, 锂脱出伴 随着氧的氧化脱出, 表现为“近水平充电平台”[16]。

$\mathrm{Li}_{2} \mathrm{MnO}_{3}$ 在活化过程中不仅会发生脱锂氧反 应、锂离子和过渡金属离子的迁移 ${ }^{[22]}$ 等，还会出现 电解液氧化分解、侵蚀正极材料等 ${ }^{[23]}$ 。Robertson 等 ${ }^{[24]}$ 认为电解液氧化分解会产生活性 $\mathrm{H}^{+}$, 而 $\mathrm{H}^{+}$会 与晶格中 $\mathrm{Li}^{+}$交换, $\mathrm{H}^{+}$含量越高，离子交换反应愈剧 烈 ${ }^{[25]}$, 促使层状构型由 $\mathrm{O} 3$ 向 $\mathrm{P} 3$ 转变, 产生 $\mathrm{O}_{2}$ 和 $\mathrm{CO}_{2}$, 而后者正是电解液分解副产物 ${ }^{[6]}$ 。

活化过程中, 脱锂氧平台随 $\mathrm{Li}_{2} \mathrm{MnO}_{3}$ 含量增加 而延长 ${ }^{[19]}$, 元素含量也会影响脱锂氧平台长度 ${ }^{[26]}$, $0.5 \mathrm{Li}_{2} \mathrm{MnO}_{3} \cdot 0.5 \mathrm{Li}\left(\mathrm{Mn}_{0.5-x} \mathrm{Ni}_{0.5-x} \mathrm{Co}_{2 x}\right) \mathrm{O}_{2}$ 中 $x=0.5$ 的材 料活化平台长度比 $x=0$ 的材料延长了近一倍 ${ }^{[5]}$ 。同 一材料, 活化程度会随着截止电压升高而加剧 ${ }^{[27]}$ 。 Ito 教授等 ${ }^{[28-31]}$ 将正极材料在 $2.0 \sim 4.5 \mathrm{~V}$ 和 $2.0 \sim 4.8 \mathrm{~V}$ 循环了 5 次, 研究材料表面区域晶相结构发现, 
2.0 4.8 V 活化后表面会形成大量缺陷; 而 $2.0 \sim 4.5 \mathrm{~V}$ 活化后, 表面会形成类针化层结构 ${ }^{[31]}$ 。另外, 温度 也会影响活化过程, 2013 年, Lanz 等 ${ }^{[32]}$ 研究发现, 材料在 $50^{\circ} \mathrm{C}$ 下活化, 电解液氧化分解等副反应比常 温条件下更为剧烈。Johnson 等 ${ }^{[33]}$ 分析认为, 温度升 高会使电解液的氧化分解加剧, 也会加速离子迁移, 加剧晶相转变程度。

分析表明, 活化过程包括不可逆脱锂氧、电解液 氧化分解和电解液对正极材料表面侵蚀等 ${ }^{[34]}$, 使材 料首次库仑效率仅为 $54 \%$ \% $73 \%{ }^{[16,24,34]}$ 。然而活化会 使部分 $\mathrm{Mn}^{4+}$ 向 $\mathrm{Mn}^{3+}$ 转变, 使正极材料具备高容量。

\section{2 循环过程中的晶相转变和电化学特性}

通常, 电极材料充电电压高于 $4.3 \mathrm{~V}$ 时 (vs $\left.\mathrm{Li}^{0}\right)$, 会出现结构中八面体中心过渡金属离子向共面四面 体中心迁移, 因而, 富锂层状氧化物在高于 $4.45 \mathrm{~V}$ 充电时会因脱锂形成大量“锂空穴”, 促使大量过渡 金属离子发生迁移 ${ }^{[35]}$ 。这些迁入离子在后续充放电 过程中会发生三种可能迁移 ${ }^{[36-38]}$ 。

(i) 四面体中心过渡金属离子迁移至锂层八面 体中心，形成低电压尖晶石晶相 ${ }^{[36,39]}$;

(ii) 四面体中心过渡金属离子迁回原八面体中 心晶位, 释放锂层八面体中心晶位, 但这一离子迁 回过程会导致滞后电压 ${ }^{[36,38,40]}$ 。

(iii) 四面体中心过渡金属离子滞留, 锂离子不 能嵌入相邻八面体中心, 使容量降低; 同时滞留离 子会阻碍锂离子传输, 使电极材料极化阻抗增大, 极化电压增大 ${ }^{[36,41]}$ 。

这三种离子迁移方式使正极材料形成低电压晶 相、出现滞后电压和极化电压增大等现象, 使正极
材料电化学性能在循环过程中持续衰减。

富锂层状氧化物正极材料在 $2.0 \sim 4.8 \mathrm{~V}$ 循环过 程中, 晶相尖晶石化转变非常显著。Mohanty 等 ${ }^{[39]}$ 利用中子粉末衍射进行深入研究发现, 四面体中心 的锂离子和锰离子含量增加, 部分四面体中心锰离 子会向锂层中迁移, 晶相结构中形成尖晶石相。这 一晶相转变的具体过程如下: 充电过程中, Li 层中 $\mathrm{Li}$ 大量脱出，使 $\mathrm{T}^{\mathrm{M}}$ 层中离子向共面四面体中心迁 移增加, 其中部分四面体中心 $\mathrm{Mn}$ 会迁移至 $\mathrm{Li}$ 层中 以维持晶相结构平衡 ${ }^{[2]}$ 。 slab $\rightarrow$ inter-slab 的 $\mathrm{Mn}$ 增 加, 使锂层中锰离子增多, 此时部分锂离子嵌入四 面体中心, 会形成尖晶石相。离子迁移随循环次数 增加而加剧, 促使尖晶石化转变区域扩大，正极材 料电化学特性随之演变(如图 3 所示)。

这一晶相转变过程在 2012 年 Francis 等 ${ }^{[23]}$ 研究 中得以证明, $\mathrm{Li}_{2} \mathrm{MnO}_{3}$ 在 $2.0 \sim 4.7 \mathrm{~V}$ 循环, 容量在 5 次循环内会递增，伴随着充放电平台下降，出现尖 晶石相特征氧化还原峰。研究发现, 这一新形成尖 晶石相氧化还原峰位于 $3.0 \mathrm{~V}$, 峰强随循环次数增加 逐渐增强 ${ }^{[43]}$ 。

滞后电压同样会影响富锂层状氧化物正极材料 的电化学性能 ${ }^{[44]}$ 。分析认为, 滞后电压是充电过程 中 $4.3 \mathrm{~V}$ 以上迁出的过渡金属离子在放电至 $3.2 \mathrm{~V}$ 时 迁回原晶格位点造成的 ${ }^{[38]}$, 滞后电压达到 $1 \mathrm{~V}$ 时, 将导致近 $10 \% \sim 15 \%$ 的锂离子不能嵌回原晶位 ${ }^{[37,45]}$ 。 研究表明, 正极材料中 $\mathrm{Li}_{2} \mathrm{MnO}_{3}$ 含量增加, $4.3 \mathrm{~V}$ 氧 化峰和 $3.2 \mathrm{~V}$ 还原峰强度增大，滞后电压增大, $50 \%$ 电荷状态下充放电曲线之间的电压差增大 ${ }^{[36]}$ 。

同时，富锂层状氧化物正极材料因晶相结构的 差异具有较大的极化阻抗，这一极化阻抗在循环过

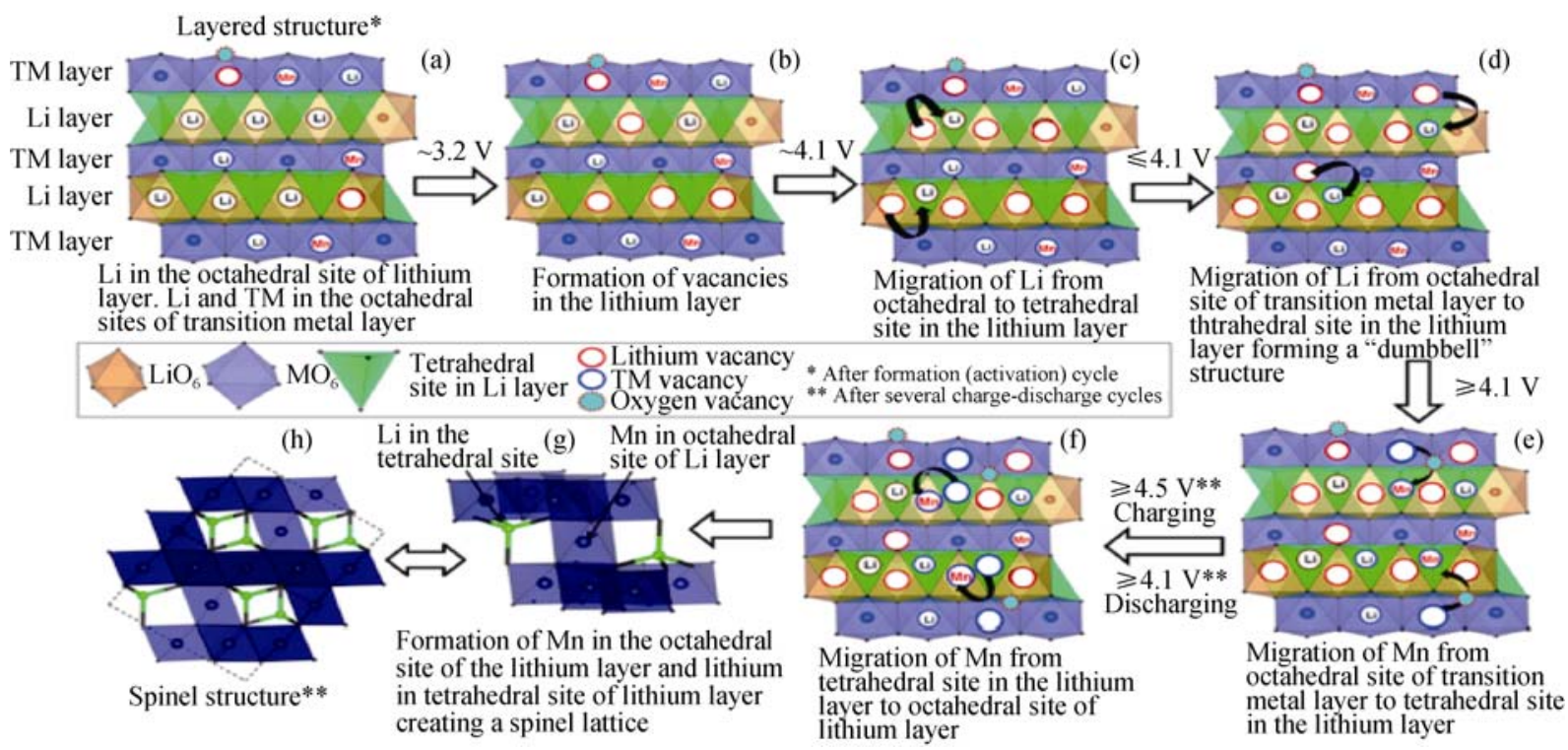

图 3 富锂固溶体层状晶相向尖晶石相转变的可能机理 ${ }^{[42]}$

Fig. 3 Proposed layered-to-spinel transformation mechanism for lithium layered cathode materials ${ }^{[42]}$ 
程中会因晶相转变逐渐增大 ${ }^{[41]}$, 使极化电压增大, 正极材料性能衰减。2014 年, Zhang 等 ${ }^{[46]}$ 研究认为, 表面阻抗增大是造成循环过程容量下降的主要原因, 内部极化阻抗增大是造成电压降的重要原因。

富锂层状氧化物正极材料电化学性能衰减是亟 待解决的关键问题, 综合活化和循环过程电化学性 能演变规律分析可知, 材料电化学性能衰减的主要 原因包括以下几个方面:

(i) 晶相不可逆转变: 四面体中心过渡金属离 子向锂层中八面体中心和过渡金属层中八面体中心 迁移的过程导致低电压尖晶石相转变和滞后电压增 大, 使电化学性能衰减 ${ }^{[45,47-48]}$;

(ii) 锂离子扩散受阻: 四面体晶位过渡金属离 子滞留, 锂离子传输受阻, 减少了锂离子可嵌入晶 位, 使极化电压增大, 锂离子脱嵌量减少 ${ }^{[49-51]}$;

(iii) 电解液的氧化与侵蚀: 高电压下, 电解液 会氧化分解并侵蚀正极材料, 使材料中活性物质溶 出和结构塌陷; 还会在材料表面形成阻碍锂离子传 输结构, 使表面阻抗增大 ${ }^{[28-30,43]}$ 。

富锂层状氧化物正极材料在 $2.0 \sim 4.8 \mathrm{~V}$ 循环过 程中, 材料表面的尖晶石化转变区域会逐渐向内发 展, 使材料出现持续容量衰减和电压下降 ${ }^{[52]}$ 。

循环过程中, 富锂正极材料中 $\mathrm{Li}_{2} \mathrm{MnO}_{3}$ 比例越 高, 晶相转变程度越大, 电压降幅越大 ${ }^{[43,53]}$, 容量 越高, 但容量保留率低 ${ }^{[19]}$ 。Liu 等 ${ }^{[51]}$ 研究发现, $\mathrm{Li}_{2} \mathrm{MnO}_{3}$ 比例为 0.3 和 0.5 时, 材料容量保留率分别 为 $59 \%$ 和 $73 \%$ 。Johnson 等 ${ }^{[33,54]}$ 通过调控 Co 含量来 优化材料循环稳定性和倍率性能。元素组成可调控 $\mathrm{Li}_{2} \mathrm{MnO}_{3}$ 比例, 改变晶相转变程度 ${ }^{[26]}$, 也可改变离 子排布特性，调控循环容量和倍率性能等 ${ }^{[19]}$ 。

合成条件方面, 烧结温度和时间会改变富锂层 状氧化物正极材料颗粒粒径和晶粒尺寸。Liu 等 ${ }^{[55]}$ 研究发现, $900{ }^{\circ} \mathrm{C}$ 合成的正极材料一次颗粒粒径为 $200 \mathrm{~nm}$, 材料电化学性能最为优异。

测试条件方面, 富锂层状氧化物正极材料在 $2.0 \sim 4.8 \mathrm{~V}$ 循环 50 次后, 容量保留率仅 $68 \%$ 。在 $2.0 \sim 4.5 \mathrm{~V}$ 进行 5 次预循环后在 $2.0 \sim 4.8 \mathrm{~V}$ 循环, 前数次充放电曲 线几乎重叠, 晶相转变减缓, 循环 50 次容量保持率提 高至 $90 \%{ }^{[29-30,56]}$ 。Seung-Ho 等 ${ }^{[57]}$ 合成的正极材料在 2.0 4.55 V 和 2.0 4.7 V 循环 50 次的容量相等, 而容 量保留率由 $88.9 \%(4.7 \mathrm{~V})$ 提高至 99\% (4.45 V)。研究 表明, 正极材料在 $25^{\circ} \mathrm{C}$ 循环时表面形成钝化层, 表面 电荷传输阻抗增大, 循环容量下降。而在 $45.4^{\circ} \mathrm{C}$ 循环 时, 锂离子扩散能力较强, 材料循环时具有更小的交 流阻抗、更高容量和容量保留率 ${ }^{[58-59]}$ 。

值得注意的是, 在循环过程中出现的电压降同
样使富锂层状氧化物正极材料的输出能量持续下 降。2012 年, Croy 等 ${ }^{[60]}$ 就开始了研究这类材料的电 压降问题, 并重点研究了 $x \mathrm{Li}_{2} \mathrm{MnO}_{3} \cdot(1-x) \mathrm{LiMO}_{2}$ 在 不同截至电压循环时滞后电压、低电压尖晶石相转 变与正极材料电压下降的关系, 发现电压降随截止 电压升高而增大 ${ }^{[37]}$ 。同时, 材料内部阻抗也会影响 电压降 ${ }^{[41]}$ 。研究表明, 合成条件和正极材料组成 ${ }^{[61]}$ 、 表面状态、Mn 化合价态 ${ }^{[62] 、}$ 材料内部元素分布 ${ }^{[63]}$ 等会改变金属离子迁移过程, 影响阻抗变化规律, 影响电化学性能演变规律。此外, 降低截止电压 ${ }^{[27]}$ 能减缓晶相转变过程, 减缓电压下降, 使正极材料 的电压降减少 ${ }^{[64]}$ 。富锂层状氧化物正极材料在低于 $4.5 \mathrm{~V}$ 循环时, 也会出现电压下降 ${ }^{[44]}$, 这一电压降的 主要原因还有待进一步研究。

\section{3 富锂层状氧化物正极材料的改性 研究}

目前, 主要通过表面改性和离子掺杂来减少和 抑制富锂层状氧化物正极材料在 $2.0 \sim 4.8 \mathrm{~V}$ 循环过 程中出现的容量衰减和电压下降。

\section{1 表面改性}

Johnson 等 ${ }^{[15]}$ 采用酸处理对 $\mathrm{Li}_{2} \mathrm{MnO}_{3}$ 进行表面 改性形成 $(1-\mathrm{z} / 2) \mathrm{Li}_{2} \mathrm{MnO}_{3} \cdot \mathrm{z} / 2 \mathrm{MnO}_{2}$ 结构, 通过 $\mathrm{MnO}_{2}$ 表面改性层来提高 $\mathrm{Li}_{2} \mathrm{MnO}_{3}$ 的循环稳定性。 Thackeray 教授等 ${ }^{[65]}$ 采用酸表面改性后的富锂层状 氧化物正极材料具有 $250 \mathrm{mAh} / \mathrm{g}$ 以上首次放电容量, 库仑效率接近 $90 \%$, 循环 10 次容量保留率为 $96 \%$ 。 这是因为正极材料表面形成了具有电化学活性 $\mathrm{MnO}_{2}$, 酸处理时间越长, 晶相衍射峰宽度增大, 出 现 3.0 2.5 V 的放电平台, $\mathrm{MnO}_{2}$ 的嵌锂平台变长, 放 电容量增加。但是, 酸处理会使正极材料形成低电 压晶相，导致放电平台降低 ${ }^{[66]}$ 。

表面副反应会使富锂层状氧化物正极材料性能 衰减 ${ }^{[16]}$ 。国内外通过对正极材料进行表面改性来抑 制表面副反应，表面改性材料可以按其电化学特性 分为电化学活性材料 ${ }^{[66-67]}$ 和电化学惰性的材料 ${ }^{[68]}$ (如 表 1 所示)。

从表 1 中可以看出, 非活性氧化物表面改性富 锂层状氧化物正极材料具有最高容量保留率, 是由 于在正极材料表面形成针化层, 有效阻隔电解液对 正极材料的侵蚀, 抑制了活性物质溶出所致, 如 $\mathrm{AlF}_{3}$ 表面改性材料表面电荷转移阻抗在循环过程中 保持稳定, 增幅不及未包覆的 $1 / 4$, 有效提高了材料 循环稳定性和倍率性能 ${ }^{[73]}$; 而 $\mathrm{FePO}_{4} 、 \mathrm{~V}_{2} \mathrm{O}_{5}$ 和 $\mathrm{MnO}_{2}$ 等活性氧化物表面改性层固有电化学特性改变了正 
表 1 富锂层状氧化物正极材料表面包覆改性研究

Table 1 Studies on coating the lithium-rich cathode materials

\begin{tabular}{llll}
\hline & \multicolumn{1}{c}{ Surface modified materials } & Initial culombical efficiency (\%) & Capacity rentention(\%) \\
\hline Unelectrochemical activity oxides & $\mathrm{Al}_{2} \mathrm{O}_{3}{ }^{[68,69]}, \mathrm{ZrO}_{2}{ }^{[69,70]}, \mathrm{ZnO}^{[69]}$, & {$[87.4,(77.1)]^{[68]}[98,96,97$,} & {$[90,84,89,84,87(60)]^{[69]}$} \\
& $\mathrm{Ta}_{2} \mathrm{O}_{5}^{[69]}, \mathrm{Nb}_{2} \mathrm{O}_{5}{ }^{[69]}$ & $96,98(92)]^{[69]}$, & $94.9(87.5)^{[70]}$ \\
Electrochemical activity oxides & $\mathrm{V}_{2} \mathrm{O}_{5}{ }^{[71]}, \mathrm{MnO}_{2}{ }^{[72]}, \mathrm{FePO}_{4}{ }^{[73]}$ & $85(79)^{[71]}, 90(83)^{[72]}$, & $90(50)^{[71]} 93(89)^{[72]}$ \\
& & $85.1-100(79)^{[73]}$ & $95(84.9)^{[73]}$ \\
Fluorides & $\mathrm{AlF}_{3}{ }^{[74]}$ & $90.8(82.8)^{[74]}$ & $91.6(73.4)^{[74]}$ \\
C Derivative & $\mathrm{C}^{[75,76]}$ & $96.7(84)^{[75]} 79.5(74)^{[76]}$ & $92(44)^{[75]}$ \\
& & & $85.1(74.7)^{[76]}$ \\
Other materials & $\mathrm{AlPO}_{4}{ }^{[68,77]}$ & $92.2(77.1)^{[68]} 78.9(66.5)^{[77]}$ & $73(65)^{[77]}, 92(71)^{[77]}$ \\
& $\mathrm{Li}_{2} \mathrm{SiO}_{3}{ }^{[77]}$ & $76.4(69.6)^{[77]}$ & \\
\hline
\end{tabular}

极材料的电化学性能。 $\mathrm{MnO}_{2}$ 改性的正极材料在 $5 / 2 C$ 倍率放电容量提高了 $29.3 \%{ }^{[72]}, \mathrm{FePO}_{4}$ 表面改 性材料在 $2 C$ 倍率放电容量提高了 $40 \%{ }^{[73]}$ 。碳包覆 可以显著提高正极材料的导电性, 改善倍率性能 ${ }^{[75]}$, 碳包覆改性正极材料在 $10 \mathrm{C}$ 倍率放电容量可达 $100 \mathrm{mAh} / \mathrm{g}^{[76]}$ 。部分正极材料表面改性富锂正极材 料同样能提高循环稳定性, 改善其倍率性能 ${ }^{[78]}$ 。富 锂层状氧化物正极材料在表面改性后首次循环不可 逆容量减少, 循环容量保留率提高, 倍率性能提高。

\section{2 离子掺杂}

富锂层状氧化物正极材料的循环稳定性还可通 过离子掺杂改性来改善, 掺杂离子主要包括非电化 学活性阳离子、电化学活性阳离子、阴离子和多离 子等四类。

\section{(1) 非电化学活性阳离子掺杂}

掺杂改性富锂正极材料的非电化学活性阳离子 主要包括 $\mathrm{Al} 、 \mathrm{Mg} 、 \mathrm{Zr} 、 \mathrm{Zn} 、 \mathrm{~K} 、 \mathrm{Na}$ 等 ${ }^{[79-87]}$ 。按离 子占位和功能可分为两类: 一类是占据 $\mathrm{Li}$ 层或 $\mathrm{TM}$ 层八面体晶格位点, 以更强键能 $\mathrm{M}=\mathrm{O}$ 提高晶相结 构稳定性, 主要有 $\mathrm{Na} 、 \mathrm{Al} 、 \mathrm{Mg} 、 \mathrm{Zr}$ 等, 如 $\mathrm{Na}$ 掺杂 容量保留率高达 $98 \%{ }^{[83]}$ 。 $\mathrm{Zr}$ 掺杂能限制晶胞体积变 化 ${ }^{[82]}, \mathrm{Al} 、 \mathrm{Mg}$ 和 $\mathrm{K}$ 等掺杂能提高锂离子扩散系数 ${ }^{[80-85]}$, 提高倍率性能; 另一类是新的晶型, 研究表明高 $\mathrm{Na}$ 掺杂改性会形成 P2 构型晶相 ${ }^{[85]}$, 两种层状构型共 存显著提高了这类正极材料的容量循环稳定性 ${ }^{[86]}$ 。

(2) 电化学活性阳离子掺杂

富锂层状氧化物正极材料改性还包括 Ni、Co、 $\mathrm{Ti} 、 \mathrm{Nb} 、 \mathrm{Ru} 、 \mathrm{Fe}$ 等电化学活性离子 ${ }^{[85-88]}$ 。Tabuchi 等 ${ }^{[87]}$ 采用 $\mathrm{Fe}$ 掺杂 $\mathrm{Li}_{2} \mathrm{MnO}_{3}$ 减少离子混排, 提高正极 材料循环稳定性。 $\mathrm{Ni}$ 掺杂是可以兼顾 $\mathrm{Li}_{2} \mathrm{MnO}_{3}$ 循环

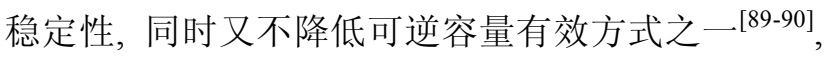
这是因为 $\mathrm{Ni}$ 具有 2 个电子电荷补偿机制, 显著提高 了可逆脱嵌锂离子 ${ }^{[91]}$, 相近的 $\mathrm{Ni}^{2+}$ 和 $\mathrm{Li}^{+}$半径可以
减少过渡金属层中的 $\mathrm{Li}^{+}$, 减缓尖晶石化转变 ${ }^{[34]}$, 除 $\mathrm{Ni}$ 以外, $\mathrm{Co} 、 \mathrm{Ti}$ 等离子掺杂也有利于提高富锂层状 氧化物正极材料的可逆循环容量 ${ }^{[92]}$ 。

此外, $\mathrm{Ru}, \mathrm{Nb}, \mathrm{Fe}$ 等也被广泛用于改性富锂层状 氧化物正极材料。在 $\mathrm{Li}_{1.2} \mathrm{Mn}_{0.6-\mathrm{x}} \mathrm{Ru}_{x} \mathrm{Ni}_{0.2} \mathrm{O}_{2}$ 中, 较高 $\mathrm{Ru}$ 掺杂量会形成 $\mathrm{Li}_{2} \mathrm{RuO}_{3}(\mathrm{P} 12 / \mathrm{m}$ 对称性)结构, $\mathrm{Ru}$ 的 $4 \mathrm{~d}$ 和 $\mathrm{O}$ 的 $2 \mathrm{p}$ 轨道交叠能有效减少不可逆氧脱出，此外，电 化学活性 $\mathrm{Ru}$ 离子掺杂也可提高材料循环容量 ${ }^{[93]}$ 。 $\mathrm{Nb}$ 掺杂改性的 $\mathrm{Li}\left[\mathrm{Li}_{0.2} \mathrm{Ni}_{0.2} \mathrm{Mn}_{0.6-x} \mathrm{Nb}_{x}\right] \mathrm{O}_{2}$ 循环 100 次容量保 持率可达 93.2\% ${ }^{[94]}$ 。 $\mathrm{Li}_{1.167} \mathrm{Ni}_{0.233} \mathrm{Co}_{0.100} \mathrm{Mn}_{0.467} \mathrm{Mo}_{0.033} \mathrm{O}_{2}$ 中引入 Mo 掺杂改性, 能稳固晶相中的 $\mathrm{O}$, 减少不可 逆脱出 $\mathrm{O}$, 这一正极材料在 $2.0 \sim 4.7 \mathrm{~V}$ 循环 50 次容量 保留率为 $88.9 \%{ }^{[57]}$ 。 Liu 等研究表明 ${ }^{[95]}, \mathrm{Fe}$ 掺杂量增 加会使不可逆脱出 $\mathrm{O}$ 增加, $\mathrm{Li}_{1.2} \mathrm{Mn}_{0.6-x / 2} \mathrm{Ni}_{0.2-x / 2} \mathrm{Fe}_{x} \mathrm{O}_{2}$ 正极材料首次库仑效率由 $83.4 \%$ 下降至 $73.6 \%$, 但 $\mathrm{Fe}$ 掺杂能提高正极材料循环容量保留率, 材料循环 80 次容量保留率高达 $90.9 \%$ 。

\section{(3) 阴离子掺杂}

阴离子掺杂改性富锂层状氧化物正极材料主要 是采用 $\mathrm{F}$ 等取代晶格中的氧, $\mathrm{F}$ 掺杂能形成部分键能 更强的 $\mathrm{F}-\mathrm{M}(\mathrm{M}=\mathrm{Ni}, \mathrm{Co}, \mathrm{Mn})$, 减少不可逆氧脱出, 提高晶体结构在活化和循环过程中的稳定性。Kang 等 ${ }^{[96]}$ 研究发现 $\mathrm{F}$ 掺杂降低了富锂层状氧化物正极材 料的表面阻抗, 故而 $\mathrm{F}$ 掺杂改性后, 材料容量在循 环过程中并未出现衰减。

聚阴离子掺杂改性富锂正极材料能提高结构中 离子的结合能, 改善循环过程中结构的稳定性, 减 缓电压衰减 ${ }^{[97]}$ 。Zhao 等 ${ }^{[98]}$ 引入 $\mathrm{PO}_{4}{ }^{3-}$ 梯度掺杂改性 富锂正极材料显著提高了正极材料离子传输性能和 容量循环稳定性(循环 100 次容量保留率 95\%)。

(5) 离子共掺杂

$\mathrm{Lim}$ 等 ${ }^{[99]}$ 采用 $\mathrm{Mg}$ 和 $\mathrm{F}$ 共掺杂改性富锂层状氧 化物正极材料, $\mathrm{Mg}$ 和 $\mathrm{F}$ 共掺杂后正极材料在循环过 
程中 $\mathrm{Mg}$ 掺杂起到稳定局部晶相结构, 而 $\mathrm{F}$ 减少氧 损失的双重功能型作用, 使正极材料循环 150 次的 容量保留率由 $86 \%$ 提高至 $90 \%$ 。

综合以上分析可以发现, 离子掺杂改性能够提 高富锂层状氧化物正极材料的容量保留率, 改善倍 率性能。但离子掺杂提高富锂层状氧化物正极材料 电化学循环稳定性的机理尚待进一步研究。

\section{4 结束语}

富锂层状氧化物正极材料具有高容量、性能可 调、价格低廉等优点, 是下一代高容量锂离子电池 重要发展体系之一。然而这类正极材料在活化后会 形成多晶相复合结构, 这一结构在后续循环过程中 会持续发生转变, 导致正极材料的电化学性能衰减, 表面改性和离子掺杂能减缓晶相转变过程, 在一定 程度上减缓和抑制电化学性能的衰减, 是改进其电 化学性能的有效途径, 但改性的机理尚待进一步研 究。要使这类正极材料满足商业化应用要求, 需要 综合考虑容量和循环稳定性, 选用适当测试条件以 确保多晶相结构循环稳定性, 兼顾最大限度地发挥 这类正极材料的容量和倍率性能。提高这类新型高 性能多晶相正极材料结构稳定性的合成和改性手段 值得进一步探索。

\section{参考文献:}

[1] ARMAND M, TARASCON J M. Building better batteries. Nature, 2008, 451(7179): 652-657.

[2] $\mathrm{YU} \mathrm{H}, \mathrm{ZHOU}$ H. High-energy cathode materials $\left(\mathrm{Li}_{2} \mathrm{MnO}_{3}-\mathrm{LiMO}_{2}\right)$ for Lithium-ion batteries. The Journal of Physical Chemistry Letters, 2013, 4: 1268-1280.

[3] GOODENOUGH J B. Cathode materials: a personal perspective. $J$. Power Sources, 2007, 174(2): 996-1000.

[4] STROBEL P, LAMBERT-ANDRON B. Crystallographic and magnetic structure of $\mathrm{Li}_{2} \mathrm{MnO}_{3}$. J. Solid State Chem., 1988, 75(1): 90-98.

[5] MASSAROTTI V, CAPSONI D, BINI M, et al. Electric and Magnetic Properties of $\mathrm{LiMn}_{2} \mathrm{O}_{4}$ - and $\mathrm{Li}_{2} \mathrm{MnO}_{3}$-type oxides. J. Solid State Chem., 1997, 131(1): 94-100.

[6] ARMSTRONG A R, HOLZAPFEL M, NOVAK P, et al. Demonstrating oxygen loss and associated structural reorganization in the lithium battery cathode $\mathrm{Li}\left[\mathrm{Ni}_{0.2} \mathrm{Li}_{0.2} \mathrm{Mn}_{0.6}\right] \mathrm{O}_{2}$. J. Am. Chem. Soc., 2006, 128(26): 8694-8698.

[7] BROUSSELY M, PERTON F, BIENSAN P, et al. $\mathrm{Li}_{x} \mathrm{NiO}_{2}$, a promising cathode for rechargeable lithium batteries. J. Power Sources, 1995, 54(1): 109-114.
[8] BOULINEAU A, CROGUENNEC L, DELMAS C, et al. Reinvestigation of $\mathrm{Li}_{2} \mathrm{MnO}_{3}$ structure: electron diffraction and High resolution TEM. Chem. Mater., 2009, 21(18): 4216-4222.

[9] XIAO R, LI H, CHEN L. Density functional investigation on $\mathrm{Li}_{2} \mathrm{MnO}_{3}$. Chem. Mater., 2012, 24(21): 4242-4251.

[10] BAREÑO J, BALASUBRAMANIAN M, KANG S H, et al. Longrange and local structure in the layered oxide $\mathrm{Li}_{1.2} \mathrm{Co}_{0.4} \mathrm{Mn}_{0.4} \mathrm{O}_{2}$. Chem. Mater., 2011, 23(8): 2039-2050.

[11] BOULinEAU A, CROGUENNEC L, DELMAS C, et al. Structure of $\mathrm{Li}_{2} \mathrm{MnO}_{3}$ with different degrees of defects. Solid State Ionics, 2010, 180(40): 1652-1659.

[12] CHEN Y F, CHEN Z X, XIE K. Effect of annealing on the first-cycle performance and reversible capabilities of lithium-rich layered oxide cathodes. The Journal of Physical Chemistry C, 2014, 118(22): 11505-11511.

[13] ZHANG QIAN, LIU WEI-WEI, FANG GUO-QING, et al. Structural and electrochemical performances of $\mathrm{Li}_{1+2 x} \mathrm{Mn}_{0.3+}{ } \mathrm{Ni}_{0.3-3 x} \mathrm{Cr}_{0.4} \mathrm{O}_{2}$ synthesized by spray-dry method. Journal of Inorganic Materials, 2013, 28(6): 616-622.

[14] NUMATA K, SAKAKI C, YAMANAKA S. Synthesis and characterization of layer structured solid solutions in the system of $\mathrm{LiCoO}_{2}-\mathrm{Li}_{2} \mathrm{MnO}_{3}$. Solid State Ionics, 1999, 117(3/4): 257-263.

[15] THACKERAY M M, JOHNSON C S, VAUGHEY J T, et al. Advances in manganese-oxide 'composite' electrodes for lithium-ion batteries. J. Mater. Chem., 2005, 15(23): 2257-2267.

[16] LEE E, KORITALA R, MILLER D J, et al. Aluminum and gallium substitution into $0.5 \mathrm{Li}_{2} \mathrm{MnO}_{3} \cdot 0.5 \mathrm{Li}\left(\mathrm{Ni}_{0.375} \mathrm{Mn}_{0.375} \mathrm{Co}_{0.25}\right) \mathrm{O}_{2}$ layered composite and the voltage fade effect. J. Electrochem. Soc., 2015, 162(3): A322-A329.

[17] JEONG J H, JIN B S, KIM W S, et al. The influence of compositional change of $0.3 \mathrm{Li}_{2} \mathrm{MnO}_{3} \cdot 0.7 \mathrm{LiMn}_{1-x} \mathrm{Ni}_{y} \mathrm{Co}_{0.1} \mathrm{O}_{2} \quad(0.2 \leqslant x \leqslant 0.5$, $y=x-0.1)$ cathode materials prepared by co-precipitation. J. Power Sources, 2011, 196(7): 3439-3442.

[18] ZHANG L, TAKADA K, OHTA N, et al. Synthesis and electrochemistry of layered $0.6 \mathrm{LiNi}_{0.5} \mathrm{Mn}_{0.5} \mathrm{O}_{2} \cdot x \mathrm{Li}_{2} \mathrm{MnO}_{3} \cdot y \mathrm{LiCoO}_{2}(x+y=$ 0.4) cathode materials. Mater. Lett., 2004, 58(25): 3197-3200.

[19] BREGER J, JIANG M, DUPRE N, et al. High-resolution X-ray diffraction, DIFFaX, NMR and first principles study of disorder in the $\mathrm{Li}_{2} \mathrm{MnO}_{3}-\mathrm{Li}\left[\mathrm{Ni}_{1 / 2} \mathrm{Mn}_{1 / 2}\right] \mathrm{O}_{2}$ solid solution. J. Solid State Chem., 2005, 178(9): 2575-2585.

[20] LU Z, BEAULIEU L Y, DONABERGER R A, et al. Synthesis, structure, and electrochemical behavior of $\mathrm{Li}\left[\mathrm{Ni}_{x} \mathrm{Li}_{1 / 3-2 \times / 3} \mathrm{Mn}_{2 / 3-x / 3}\right] \mathrm{O}_{2}$. J. Electrochem. Soc., 2002, 149(6): A778-A791.

[21] LONG B R, CROY J R, DOGAN F, et al. Effect of cooling rates on phase separation in $0.5 \mathrm{Li}_{2} \mathrm{MnO}_{3} \cdot 0.5 \mathrm{LiCoO}_{2}$ electrode materials for Li-ion batteries. Chem. Mater., 2014, 26(11): 3565-3572.

[22] KIM J S, JOHNSON C S, VAUGHEY J T, et al. Electrochemical 
and structural properties of $x \mathrm{Li}_{2} \mathrm{M}^{\prime} \mathrm{O}_{3} \cdot(1-x) \mathrm{LiMn}_{0.5} \mathrm{Ni}_{0.5} \mathrm{O}_{2}$ electrodes for lithium batteries $\left(\mathrm{M}^{\star}=\mathrm{Ti}, \mathrm{Mn}, \mathrm{Zr} ; 0 \leqslant x \leqslant 0.3\right)$. Chem. Mater., 2004, 16(10): 1996-2006.

[23] FRANCIS A S, MARKOVSKY B, SHARON D, et al. Study of the electrochemical behavior of the "inactive" $\mathrm{Li}_{2} \mathrm{MnO}_{3}$. Electrochim. Acta, 2012, 78: 32-39.

[24] ROBERTSON A D, BRUCE P G. Mechanism of electrochemical activity in $\mathrm{Li}_{2} \mathrm{MnO}_{3}$. Chem. Mater., 2003, 15(10): 1984-1992.

[25] KANG S H, KEMPGENS P, GREENBAUM S, et al. Interpreting the structural and electrochemical complexity of $0.5 \mathrm{Li}_{2} \mathrm{MnO}_{3} \cdot 0.5 \mathrm{LiMO}_{2}$ electrodes for lithium batteries $\left(\mathrm{M}=\mathrm{Mn}_{0.5-\mathrm{x}} \mathrm{Ni}_{0.5-\mathrm{x}} \mathrm{Co}_{2 x}, 0 \leqslant x \leqslant 0.5\right)$. $J$. Mater. Chem., 2007, 17(20): 2069-2077.

[26] WEI Z, XIA Y, QIU B, et al. Correlation between transition metal ion migration and the voltage ranges of electrochemical process for lithium-rich manganese-based material. J. Power Sources, 2015, 281: 7-10.

[27] ITO A, LI D, OHSAWA Y, et al. A new approach to improve the high-voltage cyclic performance of Li-rich layered cathode material by electrochemical pre-treatment. J. Power Sources, 2008, 183(1): 344-346.

[28] ITO A, LI D, SATO Y, et al. Cyclic deterioration and its improvement for $\mathrm{Li}$-rich layered cathode material $\mathrm{Li}\left[\mathrm{Ni}_{0.17} \mathrm{Li}_{0.2} \mathrm{Co}_{0.07} \mathrm{Mn}_{0.56}\right] \mathrm{O}_{2}$. J. Power Sources, 2010, 195(2): 567-573.

[29] ITO A, SATO Y, SANADA T, et al. In situ X-ray absorption spectroscopic study of Li-rich layered cathode material $\mathrm{Li}\left[\mathrm{Ni}_{0.17} \mathrm{Li}_{0.2} \mathrm{Co}_{0.07} \mathrm{Mn}_{0.56}\right] \mathrm{O}_{2}$. J. Power Sources, 2011, 196(16): 6828-6834.

[30] ZHENG J, GU M, XIAO J et al. Corrosion/fragmentation of layered composite cathode and related capacity/voltage fading during cycling process. Nano Lett., 2013, 13(8): 3824-3830.

[31] JOHNSON C S, LI N, LEFIEF C, et al. Anomalous capacity and cycling stability of $x \mathrm{Li}_{2} \mathrm{MnO}_{3} \cdot(1-x) \mathrm{LiMO}_{2}$ electrodes $(\mathrm{M}=\mathrm{Mn}, \mathrm{Ni}$, Co) in lithium batteries at $50^{\circ} \mathrm{C}$. Electrochem. Commun., 2007, 9(4): 787-795.

[32] LANZ P, VILLEVIEILLE C, NOVÁK P. Electrochemical activation of $\mathrm{Li}_{2} \mathrm{MnO}_{3}$ at elevated temperature investigated by in situ Raman microscopy. Electrochim. Acta, 2013, 109(0): 426-432.

[33] JOHNSON C S, LI N, LEFIEF C, THACKERAY M M. Anomalous capacity and cycling stability of $x \mathrm{Li}_{2} \mathrm{MnO}_{3} \cdot(1-x) \mathrm{LiMO}_{2}$ electrodes $(\mathrm{M}=\mathrm{Mn}, \mathrm{Ni}, \mathrm{Co})$ in lithium batteries at $50^{\circ} \mathrm{C}$. Electrochem. Commun., 2007, 9(4): 787-795.

[34] GREY C P, YOON W-S, REED J, et al. Electrochemical activity of li in the transition-metal sites of $\mathrm{O}_{3} \mathrm{Li}\left[\mathrm{Li}_{(1-2 x) / 3} \mathrm{Mn}_{(2-x) / 3} \mathrm{Ni}_{x}\right] \mathrm{O}_{2}$. electrochem. Solid-State Lett., 2004, 7(9): A290-A293.

[35] GU L, XIAO D, HU Y S, et al. Atomic-scale structure evolution in a quasi-equilibrated electrochemical process of electrode materials for rechargeable batteries. Adv. Mater., 2015, 27(13): 2134-2149.
[36] CROY J R, GALLAGHER K G, BALASUBRAMANIAN M, et al. Quantifying hysteresis and voltage fade in $x \mathrm{Li}_{2} \mathrm{MnO}_{3} \cdot(1-x) \mathrm{LiMn}_{0.5} \mathrm{Ni}_{0.5} \mathrm{O}_{2}$ electrodes as a function of $\mathrm{Li}_{2} \mathrm{MnO}_{3}$ content. J. Electrochem. Soc., 2014, 161(3): A318-A325.

[37] CROY J R, GALLAGHER K G, BALASUBRAMANIAN M, et al. Examining hysteresis in composite $x \mathrm{Li}_{2} \mathrm{MnO}_{3} \cdot(1-x) \mathrm{LiMO}_{2}$ cathode structures. The Journal of Physical Chemistry C, 2013, 117(13): $6525-6536$.

[38] DREYER W, GUKLKE C, HERRMANN M. Hysteresis and phase transition in many-particle storage systems. Continuum Mech. Thermodyn., 2011, 23(3): 211-231.

[39] MOHANTY D, LI J, ABRAHAM D P, et al. Unraveling the voltage-fade mechanism in high-energy-density lithium-ion batteries: origin of the tetrahedral cations for spinel conversion. Chem. Mater., 2014, 26(21): 6272-6280.

[40] DOGAN F, LONG B R, CROY J R, et al. Re-entrant lithium local environments and defect driven electrochemistry of Li-and Mnrich Li-ion battery cathodes. J. Am. Chem. Soc., 2015, 137(6): 2328-2335.

[41] LI J, CAMARDESE J, SHUNMUGASUNDARAM R, et al. Synthesis and characterization of the lithium-rich core-shell cathodes with low irreversible capacity and mitigated voltage fade. Chem. Mater., 2015, 27(9): 3366-3377.

[42] MOHANTY D, LI J, ABRAHAM D P, et al. Unraveling the voltage-fade mechanism in high-energy-density lithium-ion batteries: origin of the tetrahedral cations for spinel conversion. Chem. Mater., 2014, 26(21): 6272-6280.

[43] JOHNSON C S, LI N, LEFIEF C, et al. Synthesis, characterization and electrochemistry of lithium battery electrodes: $x \mathrm{Li}_{2} \mathrm{MnO}_{3} \cdot(1-x) \mathrm{LiMn}_{0.333} \mathrm{Ni}_{0.333} \mathrm{Co}_{0.333} \mathrm{O}_{2}(0 \leqslant x \leqslant 0.7)$. Chem. Mater., 2008, 20(19): 6095-6106.

[44] LI Y, BARENO J, BETTGE M, et al. Unexpected voltage fade in LMR-NMC oxides cycled below the "Activation" plateau. J. Electrochem. Soc., 2015, 162(1): A155-A161.

[45] GALLAGHER K G, CROY J R, BALASUBRAMANIAN M, et al. Correlating hysteresis and voltage fade in lithium- and manganese-rich layered transition-metal oxide electrodes. Electrochem. Commun., 2013, 33(0): 96-98.

[46] ZHANG X, MENG X, ELAM J W, et al. Electrochemical characterization of voltage fade of $\mathrm{Li}_{1.2} \mathrm{Ni}_{0.2} \mathrm{Mn}_{0.6} \mathrm{O}_{2}$ cathode. Solid State Ionics, 2014, 268, Part B(0): 231-235.

[47] KASAI M, NISHIMURA S, GUNJI A, et al. Electrochemical study on $x \mathrm{Li}_{2} \mathrm{MnO}_{3} \cdot(1-x) \mathrm{LiNi}_{1 / 3} \mathrm{Co}_{1 / 3} \mathrm{Mn}_{1 / 3} \mathrm{O}_{2}(x=0.5)$ layered complex cathode showing voltage hysteresis. Electrochim. Acta, 2014, 146(0): 79-88.

[48] KAN Y, HU Y, LIN C-K, et al. Migration of Mn cations in delithiated lithium manganese oxides. PCCP, 2014, 16(38): 20697- 20702. 
[49] JEONG J H, JIN B S, KIM W S, et al. The influence of compositional change of $0.3 \mathrm{Li}_{2} \mathrm{MnO}_{3} \cdot 0.7 \mathrm{LiMn}_{1-x} \mathrm{Ni}_{y} \mathrm{Co}_{0.1} \mathrm{O}_{2}(0.2 \leqslant x \leqslant 0.5$, $y=x-0.1)$ cathode materials prepared by co-precipitation. J. Power Sources, 2011, 196(7): 3439-3442.

[50] HY S, CHENG J H, LIU J Y, et al. Understanding the role of $\mathrm{Ni}$ in stabilizing the lithium-rich high-capacity cathode material $\mathrm{Li}\left[\mathrm{Ni}_{x} \mathrm{Li}_{(1-2 x) / 3} \mathrm{Mn}_{(2-x) / 3}\right] \mathrm{O}_{2}(0 \leqslant x \leqslant 0.5)$. Chem. Mater, 2014, 26(24): 6919-6927.

[51] LIU J, LIU J, WANG R, et al. Degradation and structural evolution of $x \mathrm{Li}_{2} \mathrm{MnO}_{3} \cdot(1-x) \mathrm{LiMn}_{1 / 3} \mathrm{Ni}_{1 / 3} \mathrm{Co}_{1 / 3} \mathrm{O}_{2}$ during cycling. J. Electrochem. Soc., 2014, 161(1): A160-A167.

[52] ZHENG J, XU P, GU M, et al. Structural and chemical evolution of Li- and Mn-rich layered cathode material. Chem. Mater., 2015, 27(4): 1381-1390.

[53] JOHNSON C S, KIM J S, LEFIEF C, et al. The significance of the $\mathrm{Li}_{2} \mathrm{MnO}_{3}$ component in 'composite' $x \mathrm{Li}_{2} \mathrm{MnO}_{3} \cdot(1-x) \mathrm{LiMn}_{0.5} \mathrm{Ni}_{0.5} \mathrm{O}_{2}$ electrodes. Electrochem. Commun., 2004, 6(10): 1085-1091.

[54] JOHNSON C S, KIM J S, KROPF A J, et al. Structural and electrochemical evaluation of $(1-\chi) \mathrm{Li}_{2} \mathrm{TiO}_{3} \cdot x \mathrm{LiMn}_{0.5} \mathrm{Ni}_{0.5} \mathrm{O}_{2}$ electrodes for lithium batteries. J. Power Sources, 2003, 119- 121(0): 139-144.

[55] LIU J, CHEN H, XIE J, et al. Electrochemical performance studies of Li-rich cathode materials with different primary particle sizes. $J$. Power Sources, 2014, 251(0): 208-214.

[56] ITO A, SHODA K, SATO Y, et al. Direct observation of the partial formation of a framework structure for Li-rich layered cathode material $\mathrm{Li}\left[\mathrm{Ni}_{0.17} \mathrm{Li}_{0.2} \mathrm{Co}_{0.07} \mathrm{Mn}_{0.56}\right] \mathrm{O}_{2}$ upon the first charge and discharge. J. Power Sources, 2011, 196(10): 4785-4790.

[57] YU S-H, YOON T, MUN J, et al. Continuous activation of $\mathrm{Li}_{2} \mathrm{MnO}_{3}$ component upon cycling in $\mathrm{Li}_{1.167} \mathrm{Ni}_{0.233} \mathrm{Co}_{0.100} \mathrm{Mn}_{0.467} \mathrm{Mo}_{0.033} \mathrm{O}_{2}$ cathode material for lithium ion batteries. Journal of Materials Chemistry A, 2013, 1(8): 2833-2839.

[58] YABUUCHI N, LU Y C, MANSOUR A N, et al. The influence of heat-treatment temperature on the cation distribution of $\mathrm{LiNi}_{0.5} \mathrm{Mn}_{0.5} \mathrm{O}_{2}$ and its rate capability in lithium rechargeable batteries. J. Electrochem. Soc., 2011, 158(2): A192-A200.

[59] YU C, WANG H, GUAN X, et al. Conductivity and electrochemical performance of cathode $x \mathrm{Li}_{2} \mathrm{MnO}_{3} \cdot(1-x) \mathrm{LiMn}_{1 / 3} \mathrm{Ni}_{1 / 3} \mathrm{Co}_{1 / 3} \mathrm{O}_{2}$ $(x=0.1,0.2,0.3,0.4)$ at different temperatures. J. Alloys Compd., 2013, 546(0): 239-245.

[60] CROY J R, KIM D, BALASUBRAMANIAN M, et al. Countering the Voltage decay in high capacity $x \mathrm{Li}_{2} \mathrm{MnO}_{3} \cdot(1-x) \mathrm{LiMO}_{2}$ electrodes $(\mathrm{M}=\mathrm{Mn}, \mathrm{Ni}, \mathrm{Co})$ for $\mathrm{Li}+-$ Ion batteries. J. Electrochem. Soc., 2012, 159(6): A781-A790.

[61] VU A, QIN Y, LIN C K, et al. Effect of composition on the voltage fade phenomenon in lithium-, manganese-rich $x \mathrm{Li}_{2} \mathrm{MnO}_{3} \cdot(1-x) \mathrm{LiNi}_{a} \mathrm{Mn}_{b} \mathrm{Co}_{c} \mathrm{O}_{2}$ : A combinatorial synthesis approach. J. Power Sources, 2015, 294: $711-718$
[62] VERDE M G, LIU H, CARROLL K J, et al. Effect of morphology and manganese valence on the voltage fade and capacity retention of $\mathrm{Li}\left[\mathrm{Li}_{2 / 12} \mathrm{Ni}_{3 / 12} \mathrm{Mn}_{7 / 12}\right] \mathrm{O}_{2}$. ACS Applied Materials \& Interfaces, 2014, 6(21): 18868-18877.

[63] YANG X, WANG D, YU R, et al. Suppressed capacity/voltage fading of high-capacity lithium-rich layered materials via the design of heterogeneous distribution in the composition. Journal of Materials Chemistry A, 2014, 2(11): 3899-3911.

[64] PERAlTa D, COLIN J F, BOUlineau A, et al. Role of the composition of lithium-rich layered oxide materials on the voltage decay. J. Power Sources, 2015, 280(0): 687-694.

[65] KANG S H, JOHNSON C S, VAUGHEY J T, et al. The Effects of acid treatment on the electrochemical properties of $0.5 \mathrm{Li}_{2} \mathrm{MnO}_{3} \cdot 0.5 \mathrm{LiNi}_{0.44} \mathrm{Co}_{0.25} \mathrm{Mn}_{0.31} \mathrm{O}_{2}$ electrodes in lithium cells. J. Electrochem. Soc., 2006, 153(6): A1186-A1192.

[66] KANG S H, THACKERAY M M. Enhancing the rate capability of high capacity $x \mathrm{Li}_{2} \mathrm{MnO}_{3} \cdot(1-x) \mathrm{LiMO}_{2}(\mathrm{M}=\mathrm{Mn}, \mathrm{Ni}, \mathrm{Co})$ electrodes by $\mathrm{Li}-\mathrm{Ni}-\mathrm{PO}_{4}$ treatment. Electrochem. Commun., 2009, 11(4): 748-751.

[67] WU Y, MANTHIRAM A. Effect of surface modifications on the layered solid solution cathodes $(1-z) \mathrm{Li}\left[\mathrm{Li}_{1 / 3} \mathrm{Mn}_{2 / 3}\right] \mathrm{O}_{2}-z \mathrm{Li}\left[\mathrm{Mn}_{0.5-y} \mathrm{Ni}_{0.5-y} \mathrm{Co}_{2 y}\right] \mathrm{O}_{2}$. Solid State Ionics, 2009, 180(1): 50-56.

[68] MYUNG S T, IZUMI K, KOMABA S, et al. Functionality of oxide coating for $\mathrm{Li}\left[\mathrm{Li}_{0.05} \mathrm{Ni}_{0.4} \mathrm{Co}_{0.15} \mathrm{Mn}_{0.4}\right] \mathrm{O}_{2}$ as positive electrode materials for lithium-ion secondary batteries. The Journal of Physical Chemistry C, 2007, 111(10): 4061-4067.

[69] WANG Z, LIU E, GUO L, et al. Cycle performance improvement of $\mathrm{Li}$-rich layered cathode material $\mathrm{Li}\left[\mathrm{Li}_{0.2} \mathrm{Mn}_{0.54} \mathrm{Ni}_{0.13} \mathrm{Co}_{0.13}\right] \mathrm{O}_{2}$ by $\mathrm{ZrO}_{2}$ coating. Surf. Coat. Technol., 2013, 235(0): 570-576.

[70] GAO J, KIM J, MANTHIRAM A. High capacity $\mathrm{Li}\left[\mathrm{Li}_{0.2} \mathrm{Mn}_{0.54} \mathrm{Ni}_{0.13} \mathrm{Co}_{0.13}\right] \mathrm{O}_{2}-\mathrm{V}_{2} \mathrm{O}_{5}$ composite cathodes with low irreversible capacity loss for lithium ion batteries. Electrochem. Commun., 2009, 11(1): 84-86.

[71] GUO S, YU H, LIU P, et al. Surface coating of lithium-manganese-rich layered oxides with delaminated $\mathrm{MnO}_{2}$ nanosheets as cathode materials for $\mathrm{Li}$-ion batteries. Journal of Materials Chemistry A, 2014, 2(12): 4422-4428.

[72] SUN Y K, LEE M J, YOON C S, et al. The role of $\mathrm{AlF}_{3}$ coatings in improving electrochemical cycling of Li-enriched nickel-manganese oxide electrodes for Li-ion batteries. Adv. Mater., 2012, 24(9): $1192-1196$

[73] LI ZHONG, HONG JIAN-HE, HE GANG, et al. Effect of $\mathrm{FePO}_{4}$ coating on performance of $\mathrm{Li}_{1.2} \mathrm{Mn}_{0.54} \mathrm{Ni}_{0.13} \mathrm{Co}_{0.13} \mathrm{O}_{2}$ as cathode material for Li-ion battery. Journal of Inorganic Materials, 2015, 30(2): 129-134.

[74] ZHENG J M, ZHANG Z R, WU X B, et al. The effects of $\mathrm{AlF}_{3}$ coating on the performance of $\mathrm{Li}\left[\mathrm{Li}_{0.2} \mathrm{Mn}_{0.54} \mathrm{Ni}_{0.13} \mathrm{Co}_{0.13}\right] \mathrm{O}_{2}$ positive elec- 
trode material for lithium-ion battery. J. Electrochem. Soc., 2008, 155(10): A775-A782.

[75] SONG B, ZHOU C, CHEN Y, et al. Role of carbon coating in improving electrochemical performance of $\mathrm{Li}$-rich $\mathrm{Li}\left(\mathrm{Li}_{0.2} \mathrm{Mn}_{0.54} \mathrm{Ni}_{0.13} \mathrm{Co}_{0.13}\right) \mathrm{O}_{2}$ cathode. RSC Advances, 2014, 4(83): 44244-44252.

[76] CHEN J J, LI Z D, XIANG H F, et al. Bifunctional effects of carbon coating on high-capacity $\mathrm{Li}_{1.2} \mathrm{Ni}_{0.13} \mathrm{Co}_{0.13} \mathrm{Mn}_{0.54} \mathrm{O}_{2}$ cathode for lithium-ion batteries. J. Solid State Electrochem., 2015, 19(4): 1027-1035.

[77] WU F, ZHANG X, ZHAO T, et al. Multifunctional $\mathrm{AlPO}_{4}$ coating for improving electrochemical properties of low-cost $\mathrm{Li}\left[\mathrm{Li}_{0.2} \mathrm{Fe}_{0.1} \mathrm{Ni}_{0.15} \mathrm{Mn}_{0.55}\right] \mathrm{O}_{2}$ cathode materials for lithium-ion batteries. ACS Applied Materials \& Interfaces, 2015, 7(6): 3773-3781.

[78] CHEN Y F, XIE K, ZHENG C M, et al. Enhanced Li storage performance of $\mathrm{LiNi}_{0.5} \mathrm{Mn}_{1.5} \mathrm{O}_{4}$-coated $0.4 \mathrm{Li}_{2} \mathrm{MnO}_{3} \cdot 0.6 \mathrm{LiNi}_{1 / 3} \mathrm{Co}_{1 / 3} \mathrm{Mn}_{1 / 3} \mathrm{O}_{2}$ cathode materials for Li-ion batteries. ACS Applied Materials \& Interfaces, 2014, 6(19): 16888-16894.

[79] ZHAO J, WANG Z, GUO H, et al. Synthesis and electrochemical characterization of $\mathrm{Zn}$-doped Li-rich layered $\mathrm{Li}\left[\mathrm{Li}_{0.2} \mathrm{Mn}_{0.54} \mathrm{Ni}_{0.13} \mathrm{Co}_{0.13}\right] \mathrm{O}_{2}$ cathode material. Ceram. Int., 2015, 41(9, Part A): 11396-11401.

[80] LI Q, LI G, FU C, et al. $\mathrm{K}+$-doped $\mathrm{Li}_{1.2} \mathrm{Mn}_{0.54} \mathrm{Co}_{0.13} \mathrm{Ni}_{0.13} \mathrm{O}_{2}$ : a novel cathode material with an enhanced cycling stability for lithium-ion batteries. ACS Applied Materials \& Interfaces, 2014, 6(13): 10330-10341.

[81] JIN X, XU Q, LIU H, et al. Excellent rate capability of Mg doped $\mathrm{Li}\left[\mathrm{Li}_{0.2} \mathrm{Ni}_{0.13} \mathrm{Co}_{0.13} \mathrm{Mn}_{0.54}\right] \mathrm{O}_{2}$ cathode material for lithium-ion battery. Electrochim. Acta, 2014, 136(0): 19-26.

[82] CHEN Y F, ZHENG C M, CHEN Z X. The significance of the stable Rhombohedral structure in Li-rich cathodes for lithium-ion batteries. Ionics, 2017, 23(2): 367-375.

[83] JOHNSON C S, KORTE S D, VAUGHEY J T, et al. Structural and electrochemical analysis of layered compounds from $\mathrm{Li}_{2} \mathrm{MnO}_{3}$. J. Power Sources, 1999, 81-82: 491-495.

[84] DONG X, XU Y, XIONG L, et al. Sodium substitution for partial lithium to significantly enhance the cycling stability of $\mathrm{Li}_{2} \mathrm{MnO}_{3}$ cathode material. J. Power Sources, 2013, 243: 78-87.

[85] XU J, LEE D H, CLEMENT R J, et al. Identifying the critical role of li substitution in $\mathrm{P}_{2}-\mathrm{Na}_{x}\left[\mathrm{Li}_{y} \mathrm{Ni}_{z} \mathrm{Mn}_{1-y-z}\right] \mathrm{O}_{2}(0<x, y, z<1)$ intercalation cathode materials for high-energy Na-ion batteries. Chem. Mater., 2014, 26(2): 1260-1269.

[86] YABUUCHI N, HARA R, KAJIYAMA M, et al. New $\mathrm{O}_{2} / \mathrm{P}_{2}$-type Li-excess layered manganese oxides as promising multi-functional electrode materials for rechargeable $\mathrm{Li} / \mathrm{Na}$ batteries. Advanced Energy Materials, 2015, 4(13): 13072-1-3.

[87] LEE K S, MYUNG S T, BANG H J, et al. Co-precipitation synthesis of spherical $\mathrm{Li}_{1.05} \mathrm{M}_{0.05} \mathrm{Mn}_{1.9} \mathrm{O}_{4}(\mathrm{M}=\mathrm{Ni}, \mathrm{Mg}, \mathrm{Al})$ spinel and its application for lithium secondary battery cathode. Electrochim.
Acta, 2007, 52(16): 5201-5206.

[88] KARTHIKEYAN K, AMARESH S, LEE G W, et al. Electrochemical performance of cobalt free, $\mathrm{Li}_{1.2}\left(\mathrm{Mn}_{0.32} \mathrm{Ni}_{0.32} \mathrm{Fe}_{0.16}\right) \mathrm{O}_{2}$ cathodes for lithium batteries. Electrochim. Acta, 2012, 68: $246-253$.

[89] TABUCHI M, NAKASHIMA A, ADO K, et al. The effects of preparation condition and dopant on the electrochemical property for Fe-substituted $\mathrm{Li}_{2} \mathrm{MnO}_{3}$. J. Power Sources, 2005, 146(1/2): 287-293.

[90] TABUCHI M, NAKASHIMA A, TAKEUCHI T, et al. Synthesis and electrochemical characterization of $\mathrm{Fe}$ and $\mathrm{Ni}$ substituted $\mathrm{Li}_{2} \mathrm{MnO}_{3}$-An effective means to use $\mathrm{Fe}$ for constructing "Co-free" $\mathrm{Li}_{2} \mathrm{MnO}_{3}$ based positive electrode material. J. Power Sources, 2011, 196(7): 3611-3622.

[91] LU Z, MACNEIL D D, DAHN J R. Layered cathode materials $\mathrm{Li}\left[\mathrm{Ni}_{x} \mathrm{Li}_{(1 / 3-2 x / 3)} \mathrm{Mn}_{(2 / 3-x / 3)}\right] \mathrm{O}_{2}$ for lithium-ion batteries. Electrochem. Solid-State Lett., 2001, 4(11): A191-A194.

[92] KALATHIL A K, ARUNKUMAR P, KIM D H, et al. Influence of $\mathrm{Ti}^{4+}$ on the electrochemical performance of Li-rich layered oxides high power and long cycle life of $\mathrm{Li}_{2} \mathrm{Ru}_{1-x} \mathrm{Ti}_{x} \mathrm{O}_{3}$ Cathodes. ACS Applied Materials \& Interfaces, 2015, 7(13): 7118-7128.

[93] KNIGHT J C, NANDAKUMAR P, KAN W H, et al. Effect of Ru substitution on the first charge-discharge cycle of lithium-rich layered oxides. Journal of Materials Chemistry A, 2015, 3(5): 2006 2011.

[94] LI X, XIN H, LIU Y, et al. Effect of niobium doping on the microstructure and electrochemical properties of lithium-rich layered $\mathrm{Li}\left[\mathrm{Li}_{0.2} \mathrm{Ni}_{0.2} \mathrm{Mn}_{0.6}\right] \mathrm{O}_{2}$ as cathode materials for lithium ion batteries. RSC Advances, 2015, 5(56): 45351-45358.

[95] LIU X, HUANG T, YU A. Fe doped $\mathrm{Li}_{1.2} \mathrm{Mn}_{0.6-x / 2} \mathrm{Ni}_{0.2-x / 2} \mathrm{Fe}_{x} \mathrm{O}_{2}$ $(x \leqslant 0.1)$ as cathode materials for lithium-ion batteries. Electrochim. Acta, 2014, 133(0): 555-563.

[96] KANG S H, AMINE K. Layered Li $\left(\operatorname{Li}_{0.2} \mathrm{Ni}_{0.15+0.5 z} \mathrm{Co}_{0.10} \mathrm{Mn}_{0.55-0.5 z}\right) \mathrm{O}_{2-\mathrm{z}} \mathrm{F}_{z}$ cathode materials for Li-ion secondary batteries. J. Power Sources, 2005, 146(1-2): 654-657.

[97] ZHANG H Z, LI F, Pan, GUI L, et al. The effect of polyaniondoping on the structure and electrochemical performance of Li-rich layered oxides as cathode for lithium-ion batteries. Journal of The Electrochemical Society, 2015, 162(9): A1899-A1904.

[98] ZHAO Y, LIU J T, WANG S B, et al. Surface structural transition induced by gradient polyanion-doping in Li-rich layered oxides: implications for enhanced electrochemical performance. Advanced Functional Materials, 2016, 26(26): 4760-4767.

[99] LIM S N, SEO J Y, JUNG D S, et al. The crystal structure and electrochemical performance of $\mathrm{Li}_{1.167} \mathrm{Mn}_{0.548} \mathrm{Ni}_{0.18} \mathrm{Co}_{0.105} \mathrm{O}_{2}$ composite cathodes doped and co-doped with $\mathrm{Mg}$ and F. J. Electroanal. Chem., 2015, 740(0): 88-94. 